Este libro forma parte del acervo de la Biblioteca Jurídica Virtual del Instituto de Investigaciones Jurídicas de la UNAM

\title{
COVID-19, JUSTICIA DISTRIBUTIVA, PROPIEDAD INTELEGTUAL Y GEOPOLÍTICA: UNA CONTEXTUALIZACIÓN DE LA GESTIÓN DEL GOBIERNO DE COSTA RICA ANTE LA OMS
}

\author{
Gabriela ARGUEDAS RAMÍREZ
}

SumARIO: I. Introducción. II. Contexto: la pandemia de COVID-19, un dramático impacto en los sistemas de salud en el mundo entero. III. La propuesta de Costa Rica. IV. La innovación, la colaboración y la propiedad intelectual en tiempos de pandemia. V. Antecedentes que justifican la preocupación por los usos de la propiedad intelectual en situaciones críticas. VI. Geopolitica, mercado y acceso equitativo a medicamentos en tiempos de pandemia. VII. Justicia y acceso a tecnologías en tiempos de pandemia: entre ciencia abierta y ciencia cerrada. VIII. Conclusiones

La crisis de COVID-19 deja al descubierto las fallas en el sistema de medicamentos de la manera más dolorosa posible. No es una novedad para el sur global, pero ahora los países ricos se están dando cuenta de las consecuencias de la creencia de que las fuerzas del mercado ofrecerán las soluciones biomédicas necesarias.

Ellen 't HOEN directora de Medicines Law \& Policy

\section{INTRODUCGIÓN}

En este capítulo me propongo contextualizar algunos conflictos que emergen en el contexto de la actual pandemia, entre los deberes de los Estados de responder a la crisis sanitaria y las obligaciones que deben cumplir, según 
Este libro forma parte del acervo de la Biblioteca Jurídica Virtual del Instituto de Investigaciones Jurídicas de la UNAM

las normas de propiedad intelectual que han sido establecidas por la vía de tratados internacionales y acuerdos de libre comercio. Estos conflictos, en su origen, tienen una clara relación con la aplicación del principio de la justicia distributiva. Ese aspecto fundamental lo discutiré en las conclusiones de este capítulo, con base en el análisis de la propuesta realizada por el Gobierno de Costa Rica ante la Organización Mundial de la Salud (OMS), para crear un acervo de conocimiento y tecnologías, de libre acceso para todos los países miembros de la Asamblea de la OMS.

\section{CONTEXTO: LA PANDEMIA DE COVID-19, UN DRAMÁTICO IMPACTO EN LOS SISTEMAS DE SALUD EN EL MUNDO ENTERO}

El virus del síndrome respiratorio agudo severo coronavirus 2 (SARS-CoV-2), un coronavirus emergente reconocido por primera vez en diciembre de 2019, causa la enfermedad COVID-19. Según la OMS, ${ }^{1}$ la mayoría de las personas con COVID-19 desarrollan una enfermedad leve (40\%) o moderada (40\%) aproximadamente el 15\% desarrolla una enfermedad grave que requiere apoyo de oxígeno y el 5\% tiene una enfermedad crítica.

Sobre la base de la información notificada a la OMS en virtud del Reglamento Sanitario Internacional (RSI) 2005, el Director General de la OMS declaró el 30 de enero de 2020 una emergencia de salud pública de importancia internacional y el 11 de marzo de 2020 caracterizó el brote de COVID-19 como una pandemia.

\section{LA PROPUESTA DE COSTA RICA}

Con el propósito de ampliar el acceso a productos médicos, tecnologías y datos para enfrentar la pandemia causada por el virus SARS-CoV-2, el 23 de marzo del 2020 el Gobierno de la República² envío una propuesta a la OMS para crear un mecanismo de acceso y uso de información protegida a través de diversos instrumentos de propiedad intelectual (como derechos

1 World Health Organization. Report of the WHO-China Foint Mission on Coronavirus Disease 2019 (COVID-19), s. e. febrero de 2020, disponible en: https://wrwre.who.int/docs/default-source/ coronaviruse/who-china-joint-mission-on-covid-19-final-report.pdf.

2 Bagozzi, Daniela. "WHO and Costa Rica preview technology pooling initiative to ensure access to COVID-19 health products for all". Comunicado de prensa de la OMS. 15 de mayo de 2020, disponible en: https://wrere.who.int/news-room/detail/15-05-2020-who-andcosta-rica-preview-technology-pooling-initiative-to-ensure-access-to-covid-19-health-products-for-all . 
Este libro forma parte del acervo de la Biblioteca Jurídica Virtual del Instituto de Investigaciones Jurídicas de la UNAM

de patente, modelos industriales, entre otros), necesarios en el desarrollo de medicamentos, vacunas, pruebas de diagnóstico, equipos especializados, etc. Se trata entonces de un mecanismo coordinado por la OMS, para el intercambio voluntario de conocimientos, la propiedad intelectual y los datos, y la garantía del libre acceso y uso por los países miembros de la OMS de los medicamentos y vacunas que se desarrollen. Ahondaré más en esta propuesta hacia el final del capítulo.

Esta iniciativa se presentó en medio de una pandemia inédita, que no solo ha causado una debacle en términos sanitarios, sino que, además, ha sido el telón de fondo de una crisis del multilateralismo y de la credibilidad de las instituciones gubernamentales e internacionales. A esta muy delicada situación global, se suma la creciente preocupación acerca del acceso a medicamentos y otros productos que están en desarrollo o en fase de investigación. Existen dudas fundamentadas sobre la capacidad y voluntad del modelo de negocios dominante dentro de la industria farmacéutica internacional para facilitar que las innovaciones terapéuticas estén al alcance de las grandes mayorías.

La OMS ya acogió la propuesta costarricense y se encuentra en un proceso de estudio para su aplicación. Con esta iniciativa, la OMS podría facilitar el intercambio de tecnologías financiadas por el sector público y privado, asegurando un acceso gratuito o bien la concesión de licencias en "términos razonables y asequibles, en cada país miembro". Además, el Estado costarricense también le solicitó a la OMS la creación de una base de datos de actividades de investigación y desarrollo relacionadas con los productos para atender COVID-19, incluidas las estimaciones de los costos de los ensayos clínicos y los subsidios otorgados por gobiernos y organizaciones benéficas.

La crítica situación global provocada por COVID-19 ha hecho que varios países recurran a complejos mecanismos de licencias obligatorias. Por ejemplo, hace unos días Israel ${ }^{3}$ aprobó una licencia obligatoria (es decir, levantó la exclusividad que otorga el derecho de patente) de un medicamento utilizado en pacientes $\mathrm{VIH}+$, pero que parece tener también actividad terapéutica en la infección por SARS-CoV-2 (coronavirus). De esta manera, el Estado de Israel no se ve obligado a pagar las altas sumas que la casa fabricante podría exigir por este medicamento, para ser utilizado como opción de tratamiento en esta pandemia.

3 Silverman (ed.), "AbbVie will allow generic copies of its HIV pill in Israel after the government approved a license", STAT, 20 de marzo de 2020, disponible en: https://wrere. statnews.com/pharmalot/2020/03/20/abbvie-israel-hiv-kaletra-coronavirus-covid19/. 
Este libro forma parte del acervo de la Biblioteca Jurídica Virtual del Instituto de Investigaciones Jurídicas de la UNAM

La gestión del gobierno costarricense se suma a una larga historia de luchas sociales para presionar por la concesión de licencias obligatorias para medicamentos indispensables para diversos grupos de pacientes, pero cuyo precio es prohibitivo. Recordemos que las normas establecidas en la Organización Mundial del Comercio (OMG) para este tipo de licencias quedaron aún más restringidas en razón de los términos dispuestos en los acuerdos bilaterales de libre comercio que Estados Unidos (EEUU) ha firmado con varios países, incluyendo el North American Free Trade Agreement (NAFTA) y el Central American Free Trade Agreement (CAFTA).

Pero la propuesta costarricense de crear un acervo de derechos de propiedad intelectual de libre acceso para todos los países va mucho más allá del mecanismo de licencia obligatoria. El objetivo es atraer una amplia gama de tecnologías y una mayor cantidad de entidades, en lugar de aplicar el engorroso proceso de disputa por una licencia obligatoria, caso por caso (es decir, para cada medicamento o tecnología diagnóstica, etcétera).

Esta estrategia del gobierno apuesta por conseguir, no solo para Costa Rica, sino para todos los países, un acceso más equitativo y articulado a productos indispensables para atender la pandemia. Lo más significativo de esta acción es que abre la oportunidad, en el terreno internacional, para ponerle límites (al menos en situaciones extremas como una pandemia) al enorme poder de negociación que tienen las grandes corporaciones farmacéuticas. En este proceso, también permite volver a colocar el análisis crítico del modelo de innovación y desarrollo dominante en el campo farmacéutico, en el centro de las discusiones sobre el derecho humano a la salud y el precio de los medicamentos.

Para comprender con más detalle la importancia de esta propuesta y cómo ejemplifica la aplicación del principio de justicia distributiva más allá de la discusión sobre la asignación de recursos escasos en una pandemia, es preciso explicar, aunque sea de manera somera, el papel que tiene la propiedad intelectual en esta crisis.

\section{LA INNOVACIÓN, LA COLABORACIÓN Y LA PROPIEDAD INTELEGTUAL EN TIEMPOS DE PANDEMIA}

La crisis global desencadenada por esta pandemia ha demandado de todos los países una respuesta extraordinariamente urgente, que ha requerido de la suma de esfuerzos en todo el mundo para construir soluciones lo más rápidamente posible. Múltiples acciones colaborativas en investigación, desarrollo tecnológico e innovación están en curso para apoyar y fortalecer los sistemas 
Este libro forma parte del acervo de la Biblioteca Jurídica Virtual del Instituto de Investigaciones Jurídicas de la UNAM

sanitarios: desde sistemas para aumentar la capacidad de fabricación de equipos de protección personal (EPP), pasando por la innovación para elaborar más y más rápidas pruebas de diagnóstico, hasta la investigación clínica para comprobar la seguridad y eficacia de vacunas experimentales. El mundo entero está siendo testigo de cuán necesarios y vitales son, para la supervivencia de nuestra especie, los sistemas de investigación y desarrollo.

Un grupo de investigadores e investigadoras de la Universidad de Cambridge, en su working paper titulado Crisis-Critical Intellectual Property: Findings from the COVID-19 Pandemic, ${ }^{4}$ identificó una serie de sectores tecnocientíficos críticos indispensables para responder a la pandemia. El objetivo de los autores de este estudio es crear conciencia de que las consideraciones de propiedad intelectual deben abordarse temprano durante una pandemia, para evitar conflictos legales que podrían obstaculizar el acceso a tecnologías vitales en la respuesta una crisis sanitaria como la que estamos viviendo.

Como bien indican los autores, los numerosos esfuerzos para contener la pandemia lo más rápido posible requieren tomar en consideración los múltiples, diversos y complejos aspectos de propiedad intelectual relevantes en tres áreas críticas para enfrentar la pandemia de COVID-19: prevención de una mayor propagación, incluido el desarrollo de vacunas, el desarrollo de diagnósticos y el desarrollo de medicamentos efectivos y seguros para tratar la enfermedad (sobre todo, las manifestaciones más severas).

Los eventuales conflictos políticos y jurídicos respecto a derechos de propiedad intelectual asociados a sectores tecnocientíficos críticos en la atención de la pandemia deben preverse con suficiente antelación, porque demandan la toma de decisiones tanto internacionales como nacionales para asegurar que dichos posibles conflictos no se presenten o bien puedan resolverse rápidamente. De lo contrario, las disputas legales y geopolíticas por derechos de patente, secretos industriales, derechos sobre modelos industriales, etc., podrían provocar enormes atrasos en el desarrollo, escalamiento, manufactura y distribución de los diversos bienes y servicios indispensables para controlar y erradicar la pandemia. Además, dichos conflictos podrían también encarecer los productos finales e incluso, podrían obstaculizar totalmente la producción de conocimiento y la innovación necesaria para crear las soluciones tecnológicas que la actual crisis nos demanda.

El conjunto de propiedad intelectual, relevante para la investigación, desarrollo, fabricación y distribución de productos, servicios y tecnologías críticos para enfrentar la actual pandemia incluye desde propiedad inte-

4 Tietze, Frank et al., "Crisis-Critical Intellectual Property: Findings from the COVID-19 Pandemic", Centre for Technology Management Working Paper Series, núm. 2, abril de 2020, disponible en: https://papers.ssrn.com/sol3/papers.cfm?abstract_id=3569282. 
Este libro forma parte del acervo de la Biblioteca Jurídica Virtual del Instituto de Investigaciones Jurídicas de la UNAM

lectual formal y registrada, como patentes, derechos de diseño, marcas comerciales, propiedad intelectual formal no registrada, derechos de autor, diseños industriales, secretos comerciales para los procesos de fabricación, así como know-how.

De acuerdo al Coordinated Global Research Roadmap de la OMS ${ }^{5}$ se han identificado, como mínimo, las siguientes prioridades globales en investigación y desarrollo:

FASE 1. Definir las prioridades de investigación:

- Hoja de ruta de investigación global con financiación coordinada

- Herramientas y protocolos de investigación robustos

- Priorización basada en evidencia de $\mathrm{MCM}^{6}$ experimentales para evaluar

- Intercambio rápido de datos y muestras al tiempo que se garantiza de forma justa y equitativa acceso a beneficios

FASE 2. Facilitar acciones de investigación coordinadas

- Centrarse en la investigación que puede salvar vidas ahora.

- Acceso rápido a intervenciones experimentales "prometedoras" a través de aprobación de emergencia o Acceso ampliado (si no es posible realizar aprobación de emergencia)

- Uso de protocolos genéricos para acelerar la acumulación de evidencia sólida

- Intercambio rápido de datos y muestras al tiempo que se garantiza de forma justa y equitativa acceso a beneficios

FASE 3. Ampliar la producción de innovaciones que han superado un "criterio de aprobación" acordado

- Ampliación de la tecnología y enfoques de ampliación rentables

- Evaluaciones económicas independientes de mercado y acceso

- Consideración de las innovaciones con verdadero potencial de escalamiento

5 Organización Mundial de la Salud, A Coordinated Global Research Roadmap: 2019 Novel Coronavirus, s. e. Suiza, marzo de 2020, p. 11.

6 Por las siglas en inglés de Medical countermeasures: son aquellos productos biológicos, medicamentos o equipo, aprobado por la FDA, que pueden ser utilizados en una emergencia o potencial emergencia de salud pública 
Este libro forma parte del acervo de la Biblioteca Jurídica Virtual del Instituto de Investigaciones Jurídicas de la UNAM

En muchos países se han creado alianzas estratégicas entre empresas farmacéuticas, de biotecnología y universidades para dar respuesta a estos desafíos. Ya están en curso numerosos proyectos de investigación para desarrollar vacunas y tratamientos, y para explorar segundos usos de fármacos existentes que podrían tener una acción terapéutica en COVID-19. También se han conformado consorcios entre grandes compañías farmacéuticas como el que se creó entre Novartis, Bristol-Myers Squibb y GSK. ${ }^{7}$

Empresas de sectores no vinculados al ámbito sanitario han reorganizado sus líneas de producción para contribuir con la producción de productos necesarios en los hospitales, que están escaseando debido a la alta demanda. Por ejemplo, el desafío de la falta de ventiladores del Reino Unido sumó en un consorcio a Airbus, GKN, Roll-Royce, Siemens, grupo Smiths. ${ }^{8}$ Una gran diversidad de grupos autoconvocados de personal científico y de ingeniería se han sumado a la tarea de diseñar y producir protectores faciales y otros equipos. ${ }^{9}$

El campo de las ciencias informáticas también ha producido innovaciones muy rápidamente para apoyar las labores de prevención, diagnóstico y tratamiento. Usando plataformas de datos abiertos, desarrollando modelos epidemiológicos y otros sistemas de proyección ofrecen información clave para la toma de decisiones de políticas de salud pública. ${ }^{10}$

Muchos de estos sectores, fundamentales para poder controlar la pandemia y mitigar sus efectos, son usuarios intensivos de los mecanismos jurídicos de protección de la propiedad intelectual. De ahí que algunas otras

7 "Novartis partners with life sciences companies to fight Covid-19Pharmaceutical", Technology News, 27 de marzo de 2020, disponible en: https://wrere.pharmaceutical-technology. com/news/novartis-partners-companies-covid-19/; "What are pharmaceutical companies doing to tackle COVID-19?", The Association of the British Pharmaceutical Industry, s. f, disponible en: https://wwre.abpi.org.uk/medicine-discovery/covid-19/what-are-pharmaceutical-companies-doing-totackle-the-disease/; McGrail, S., "NIH Ties Up with Top Pharma Companies for COVID-19 Drug Discovery". Pharmanews Intelligence, 20 de abril de 2020, disponible en: https://pharmanewesintel.com/news/nih-ties-up-with-top-pharma-companies-for-covid-19-drug-discovery.

8 Davies, Rob. "Ventilator Challenge UK to start production in Covid-19 fight", The Guardian, 29 de marzo de 2020, disponible en: https://wwre.theguardian.com/world/2020/mar/ 29/ventilator-challenge-uk-to-start-production-in-covid-19-fight.

9 Statt, Nick, "3D printers are on the front lines of the covid-19 pandemic", The Verge, 25 de mayo del 2020, disponible en: https://wrew.theverge.com/2020/5/25/21264243/faceshields-diy-ppe-3d-printing-coronavirus-covid-maker-response; Cámara de Industrias de Costa Rica, Iniciativa interinstitucional apoya a CCSS en la producción local de equipo de protección personal (EPP), 13 de abril del 2020, disponible en: https://cicrcom/iniciativa-interinstitucional-apoya-a-ccss-en-laproduccion-local-de-equipo-de-proteccion-personal-epp/.

10 Moradian, N. et al., "The urgent need for integrated science to fight COVID-19 pandemic and beyond", fournal of Translational Medicine, núm. 205, 2020, disponible en: https:// translational-medicine.biomedcentral.com/articles/10.1186/s12967-020-02364-2. 
Este libro forma parte del acervo de la Biblioteca Jurídica Virtual del Instituto de Investigaciones Jurídicas de la UNAM

organizaciones médicas y de derechos humanos estén manifestando su preocupación respecto a los posibles obstáculos que puedan surgir si estos sectores exigen a los Estados el cumplimiento de las condiciones que ellos determinen para autorizar el uso de su propiedad intelectual. Incluso ya algunos gobiernos (Chile, Canadá e Israel) han aprobado el uso de licencias obligatorias para ciertos productos farmacéuticos. ${ }^{11}$

\section{ANTEGEDENTES QUE JUSTIFICAN LA PREOGUPACIÓN POR LOS USOS DE LA PROPIEDAD INTELEGTUAL EN SITUACIONES CRÍTICAS}

La estrategia de armonizar las normas de propiedad intelectual a través de tratados de derecho internacional, que imponen obligaciones a los países, ha sido cuestionada porque ha afectado de manera desproporcionada a los países más pobres. Además, el objetivo prioritario no es armonizar la normativa para que más empresas, en una cantidad mayor de países, puedan entrar a más mercados; sino que las normas de propiedad intelectual vinculadas al comercio internacional faciliten la maximización de ganancias para las corporaciones más grandes, a costa de las necesidades de la población más vulnerabilizada.

Por ejemplo, la protección vía patente de invención de medicamentos esenciales, aumenta los precios e impide la competencia y la generación de capacidad de manufactura local. Sobre este problema se ha investigado ampliamente, desde hace al menos 20 años. Una síntesis sobre los conflictos más importantes al respecto la podemos encontrar en el informe del High Level Panel UN Access Medicines, del $2016 .{ }^{12}$

De acuerdo con la visión de la Agenda $2030^{13}$ y una recomendación de la Comisión Global sobre el VIH y el mandato de Naciones Unidas para que el Secretario General estableciera un organismo de alto nivel para

11 Houldsworth, A., "The key covid-19 compulsory licensing developments so far", IAM, 7 de abril de 2020, disponible en: https://wwre.iam-media.com/coronavirus/the-key-covid-19-compulsory-licensing-developments-so-far; Green, A., "COVID-19: Countries race to strengthen compulsory licensing legislation", Devex, 30 de junio de 2020, disponible en: https://wrere.devex. com/news/covid-19-countries-race-to-strengthen-compulsory-licensing-legislation-97595.

12 Dreifuss, R. y Gontebanye, F. (eds.), Report of the United Nations Secretary-General's HighLevel Panel On Access to Medicines, ONU, septiembre de 2016, disponible en: http://wrere.unsgaccessmeds.org/final-report.

13 Asamblea General de las Naciones Unidas, Resolución aprobada por la Asamblea General el 25 de septiembre de 2015. Transformar nuestro mundo: la Agenda 2030 para el Desarrollo Sostenible, Suiza, 2015, disponible en: https://wrere.un.org/ga/search/view_doc.asp?symbol=A/RES/ $70 / 1$ ELLang $=S$. 
Este libro forma parte del acervo de la Biblioteca Jurídica Virtual del Instituto de Investigaciones Jurídicas de la UNAM

proponer formas de incentivar la innovación en tecnologías de la salud y aumentar el acceso a medicamentos y tratamientos, Ban Ki-moon anunció en 2015, el nombramiento de un Panel de Alto Nivel sobre Innovación y Acceso a Tecnologías Sanitarias.

Ese panel de alto nivel fundamentó su trabajo en el compromiso de los Estados miembros de las Naciones Unidas de "remediar la incoherencia entre políticas que protegen los derechos justificables de los inventores, el derecho internacional de los derechos humanos, las normas comerciales y la salud pública en el contexto de las tecnologías sanitarias". ${ }^{14}$ Las incoherencias a las que el informe hace referencia tienen que ver con la salud pública y el choque entre normas del derecho internacional de los derechos humanos internacionales con las normas del comercio internacional relacionadas con derechos de propiedad intelectual.

Todos esos tratados internacionales, convenciones y acuerdos tienen diferentes objetivos y son adoptados por los países en diferentes momentos. Los Estados tienen la obligación de proteger y cumplir con el derecho a la salud que tienen los habitantes y esto requiere, entre otras medidas, garantizar el acceso de la población a los medicamentos, y en particular a los medicamentos esenciales, como puede ser, por ejemplo, una vacuna en un contexto como el actual.

Por otro lado, los Estados también deben acatar las normas establecidas en los acuerdos comerciales y en los tratados internacionales sobre propiedad intelectual. Sin embargo, el imperativo de respetar las patentes sobre tecnologías sanitarias podría, en ciertos casos, crear obstáculos a los objetivos de salud pública.

La adopción del Acuerdo de la OMC sobre los Aspectos de los Derechos de Propiedad Intelectual relacionados con el Comercio (ADPIC) en 1994 creó un nuevo estándar de protección y aplicación de la propiedad intelectual, a nivel global, que redefinió las reglas del juego del mercado mundial. Posteriormente a la firma de los ADPIC, inició un intenso proceso de negociación y firma de acuerdos de libre comercio que impusieron obligaciones aún más amplias con respecto a los derechos de patentes y datos de prueba sobre tecnologías sanitarias, sobrepasando lo establecido en los ADPIC. Esas normativas se conocen como disposiciones ADPIC-plus (en inglés, TRIPS-plus), y han limitado de manera significativa el acceso a medicamentos y otras tecnologías sanitarias. ${ }^{15}$ Debe tenerse en consideración

14 Dreifuss y Gontebanye, op. cit., p. 3. Traducción propia.

15 Arguedas Ramírez, G., "Problemas éticos asociados al patentamiento de genes humanos". Tesis sometida a la consideración de la Comisión del Programa de Estudios de 
Este libro forma parte del acervo de la Biblioteca Jurídica Virtual del Instituto de Investigaciones Jurídicas de la UNAM

que estas son normas vigentes en los países que tienen tratados de libre comercio con EEUU y que determinarían los términos de ingreso de nuevos medicamentos o vacunas para COVID19, si no existe de previo algún acuerdo para exceptuar que se otorguen derechos de propiedad intelectual a esas nuevas tecnologías.

En el año 2009, Anand Grover, ${ }^{16}$ en calidad de Relator Especial de Naciones Unidas para el derecho humano a la salud, presentó ante la Asamblea General de Naciones Unidas su informe A/HRC/11/12, en el cual analizó el impacto de las normas ADPIC-plus en el ámbito del derecho de patentes en los acuerdos de libre comercio. Si bien las disposiciones ADPIC-plus en los tratados de libre comercio difieren de un acuerdo a otro, sus propósitos son, en general, los siguientes:

- Ampliar el plazo de la patente

- Introducir la exclusividad de datos

- Introducir el vínculo de la patente con el registro y la aprobación de medicamentos

- Crear nuevos mecanismos de protección de los derechos de propiedad intelectual

Todas estas estrategias entorpecen el ingreso de medicamentos genéricos y hacen engorrosa, lenta y a veces, imposibles de aplicar, las escasas excepciones o flexibilidades que los Estados tienen para levantar esos derechos de las compañías farmacéuticas, cuando existe una imperiosa necesidad de salud pública que requiere la producción o importación de un medicamento o alguna otra tecnología sanitaria.

Como lo expone claramente el Panel de Alto Nivel, ${ }^{17}$ la innovación y el acceso a las tecnologías sanitarias son procesos complejos y multidimensionales, de alcance global. Los problemas y obstáculos que el sistema global de $\mathrm{I}+\mathrm{D}+\mathrm{i}$ (investigación, desarrollo e innovación) en medicamentos y otras tecnologías médicas tiene para responder a las necesidades globales y, sobre todo, a las necesidades de las mayorías más vulnerabilizadas está profundamente ligado con el funcionamiento del sistema global es la PI.

Posgrado de la Maestría Interinstitucional en Bioética para optar por al grado y título de Maestría Académica en Bioética, Universidad de Costa Rica, 2011.

16 Report of the Special Rapporteur on the right of everyone to the enjoyment of the highest attainable standard of physical and mental health, Anand Grover, A/HRC/11/12, Asamblea General de las Naciones Unidas, 31 de marzo de 2009.

17 Dreifuss y Gontebanye, op. cit., passim. 
Este libro forma parte del acervo de la Biblioteca Jurídica Virtual del Instituto de Investigaciones Jurídicas de la UNAM

A pesar del notable avance en ciencia y tecnología (financiado en buena parte a través de fondos públicos), ${ }^{18}$ millones de personas continúan sufriendo y muriendo de condiciones tratables debido a la falta de acceso a tecnologías sanitarias. Según el informe del Panel de Alto Nivel sobre acceso a medicamentos:

En algunos casos, la causa radica en la insuficiencia de recursos de I+D para enfermedades en las que el mercado no ofrece suficiente retorno de la inversión. Los antibióticos suelen ofrecer poca recompensa pecuniaria por años de investigación a menudo costosa. En estas circunstancias, los expertos advierten que los virus, bacterias, parásitos y hongos resistentes a los medicamentos podrían causar 10 millones de muertes al año en todo el mundo para 2050. El modelo actual de innovación médica está mal equipado para responder a la creciente aparición de enfermedades infecciosas, como Ébola y Zika. Mientras tanto, las enfermedades tropicales desatendidas (ETD) siguen recibiendo financiación inadecuada para $\mathrm{I}+\mathrm{D}$, a pesar de que más de mil millones de personas viven con una o más ETD. ${ }^{19}$

A inicios de este año, la OMS, la OMPI y la OMC publicaron la segunda edición del informe Promoting Access to Medical Technologies and Innovation Intersections between Public Health, Intellectual Property and Trade. ${ }^{20}$ Según explican los directores de estas tres instancias intergubernamentales en el prólogo de este documento, la primera edición del estudio se publicó en 2012 con el objetivo de apoyar la cooperación internacional en cuestiones de salud, propiedad intelectual y comercio a través de una plataforma para compartir la experiencia práctica y la comprensión de una amplia gama de instrumentos de política. La versión del 2020 se concibe además como un medio para apoyar, e informar sobre, la cooperación técnica y los debates políticos en un momento en que el mundo se enfrenta a los desafíos multidimensionales de la respuesta a la pandemia de COVID-19. Para los directores de la OMS, OMPI y OMC, este estudio debe ayudar en el proceso necesario para lograr el acceso universal a las tecnologías médicas esenciales y fortalecer y diversificar los sistemas de innovación para responder a la demanda en evolución.

18 Mazzucato, M. y Semieniuk, G., "Public financing of innovation: new questions", $O x$ ford Review of Economic Policy, vol. 33, núm. 1, 2017, pp. 24-48; Nayak, R. et al., "Public sector financial support for late stage discovery of new drugs in the United States: cohort study", British Medical Fournal, vol. 367, núm. 15766, 2019.

19 Dreifuss y Gontebanye, op. cit., p. 7. Traducción propia.

20 WTO, WHO, WIPO. Promoting Access to Medical Technologies and Innovation Intersections between Public Health, Intellectual Property and Trade. 2a. ed., Ginebra, 2020. 
Este libro forma parte del acervo de la Biblioteca Jurídica Virtual del Instituto de Investigaciones Jurídicas de la UNAM

Este documento toma como base una serie de resoluciones de la Asamblea General de las Naciones Unidas emitidas en el contexto de la actual crisis global por COVID-19, a saber: A/RES/74/270 "Solidaridad mundial para combatir la enfermedad del coronavirus 2019 (COVID-19)", A/ RES/74/274 "Cooperación internacional para garantizar el acceso mundial a medicamentos, vacunas y equipos médicos para enfrentar COVID-19", en conjunto con la resolución WHA73.1 "Respuesta COVID-19", de la Asamblea Mundial de la Salud.

A través de estas resoluciones, estos organismos internacionales y los Estados que los conforman, reconocieron el impacto dramático que la pandemia ha tenido en los sistemas de salud en casi todo el mundo. En algunos casos, la crisis ha sobrepasado por completo la capacidad existente, resultando en un alto número de muertes que podrían haberse evitado si las condiciones materiales mínimas hubiesen existido. La tragedia humana que se ha vivido a nivel global ha puesto en evidencia la innegable necesidad de cooperación y colaboración entre las naciones y los diversos sectores sociales.

En el informe citado, la OMS, la OMPI y la OMC concluyen que esta extraordinaria amenaza para la salud y los medios de vida de las personas requiere de una intensa cooperación internacional para que así los Estados puedan: fortalecer y ampliar la infraestructura necesaria de sus sistemas de salud; asegurar la disponibilidad de productos, tecnologías y equipo de protección (tarea que se torna mucho más difícil para los países más empobrecidos y que empeora conforme las líneas de producción no logran suministrar a tiempo las demandas crecientes); impulsar y sostener los procesos necesarios para el desarrollo, fabricación y distribución equitativa de pruebas diagnósticas, vacunas, medicamentos, dispositivos médicos y otras tecnologías relevantes.

\section{GEOPOLÍTICA, MERCADO Y ACGESO EQUITATIVO A MEDICAMENTOS EN TIEMPOS DE PANDEMIA}

A pesar de la diplomacia de los Estados en los foros de las instituciones intergubernamentales y de las declaraciones emitidas tanto desde la ONU, como desde la OMC y la OMS, los conflictos relacionados con aspectos de la propiedad intelectual, en el contexto de la pandemia, han comenzado a surgir.

Veamos el caso de lo que sucedió en los Países Bajos. Un reactivo de Roche, necesario para procesar pruebas de diagnóstico de infección por SARS-CoV-2, comenzó a escasear y ante la negativa inicial de Roche de hacer pública la formulación del buffer para que otros fabricantes pudieran 
Este libro forma parte del acervo de la Biblioteca Jurídica Virtual del Instituto de Investigaciones Jurídicas de la UNAM

producirlo, el gobierno de los Países Bajos inició una investigación sobre la posición de Roche con respecto a los equipos y materiales de prueba de COVID-19. Finalmente, se llegó a un acuerdo y Roche se comprometió a divulgar todos los conocimientos técnicos pertinentes y a aumentar la producción para mejorar las capacidades procesamiento de pruebas. ${ }^{21}$

Quizás sea la discusión sobre el acceso a las posibles vacunas el ejemplo más claro y preocupante de los efectos que puede llegar a tener un conflicto legal asociado a una patente sobre un medicamento. Aunque las vacunas para COVID-19 aún se encuentran en el proceso de investigación clínica para comprobar seguridad y eficacia, varios Estados han realizado millonarias inversiones para garantizar que la disponibilidad de capacidad de fabricación sea suficiente para producir los altos volúmenes necesarios para cubrir la demanda global. Al respecto surgen varias preocupaciones.

Una de esas preocupaciones, y quizás la más importante en el actual contexto, gira en torno a la producción y distribución de la potencial vacuna contra el SARS-GoV-2. Gavi, la alianza global para la producción de vacunas, lazó un nuevo fondo multimillonario, el Gavi Covax Advance Market Commitment (AMC), para subsidiar las vacunas contra la infección por SARS-CoV-2 (cuando lleguen a estar disponibles) para países de bajos ingresos. El fondo tiene como objetivo acelerar la fabricación de una vacuna COVID-19 a gran escala y distribuirla según la necesidad, en lugar de la capacidad de pago. El Gavi Covax AMG se inspira en un fondo similar para vacunas neumocóccicas que Gavi ha gestionado desde 2008. ${ }^{22}$

El funcionamiento del fondo de Gavi para la vacuna contra el neumococo recibió críticas de importantes organizaciones como Médicos Sin Fronteras (MSF). MSF sostiene que el fondo aceptó la presión de las compañías farmacéuticas sobre el precio de las vacunas. Con base en esa experiencia previa afirmó que: "Los gobiernos y Gavi deben exigir que las corporaciones farmacéuticas abran los libros para que podamos ver cuánto costará realmente producir las vacunas COVID-19 potenciales". ${ }^{23}$

Además del fondo Gavi, se han lanzado otros fondos conjuntos, como el Inclusive Vaccine Alliance, en el que participan Italia, Francia, Alemania y

21 "Roche says it has provided Dutch with key corona test recipe", Dutch Newes, 28 de marzo de 2020, disponible en: https://wwre.dutchnews.nl/news/2020/03/roche-says-it-has-provided-dutch-with-key-corona-test-recipe/.

22 Usher, A., "COVID-19 vaccines for all? If an effective COVID-19 vaccine is developed, how will it get to everyone in need?", The Lancet, vol. 395, 13 de junio de 2020.

23 "Any future COVID-19 vaccines must be sold at cost and accessible to all". Comunicado de prensa. MSF. 3 de junio de 2020, disponible en: https://wrere.msforg/any-future-covid19-vaccines-must-be-sold-cost. 
Este libro forma parte del acervo de la Biblioteca Jurídica Virtual del Instituto de Investigaciones Jurídicas de la UNAM

los Países Bajos, que tiene como objetivo establecer una base de fabricación europea de vacunas COVID-19 para la UE y otros países. Por otra parte, está la propuesta presentada por el gobierno de Costa Rica para crear Fondo de Acceso a la Tecnología COVID-19, el cual fue adoptado por la OMS y ha sido respaldado por más de 30 países de ingresos medios. Sin embargo, solo cuatro países de ingresos altos han dado su adhesión a este plan: Noruega, los Países Bajos, Luxemburgo y Portugal. Como se explicó anteriormente, la propuesta de Costa Rica ofrece un marco ético-político para una asignación equitativa global de los diversos recursos diagnósticos y terapéuticos indispensables para paliar esta pandemia.

El Premio Nobel en economía y creador del concepto Advance Market Commitment, Michael Kremer, ${ }^{24}$ ha manifestado su preocupación de que la cooperación internacional en el desarrollo de vacunas pueda fracasar debido al "nacionalismo de las vacunas". Las más grandes potencias económicas del mundo, EEUU y China, no están participando de las iniciativas internacionales para financiar la investigación, desarrollo y manufactura de la potencial vacuna o vacunas, sino que han apostado por invertir en la investigación de empresas radicadas en esos países con el objetivo prioritario de atender sus respectivos mercados nacionales. EEUU, además, rompió relaciones con la OMS y no participó en el evento de lanzamiento de la iniciativa Coalition for Epidemic Preparedness Innovations (CEPI)-COVID-19, respaldada por la OMS para recaudar $\$ 8$ mil millones para el desarrollo de vacunas y medicamentos para tratar la enfermedad COVID-19. China tampoco iba a participar, pero atendió la reunión en el último minuto, de manera sorpresiva. ${ }^{25}$

A pesar de que CEPI es un coalición más abierta y menos comprometida con agendas que podrían derivar en el "nacionalismo de las vacunas", persisten dudas y críticas acerca de cuán claro es el compromiso de la organización con principios como el de la justicia distributiva. Antes de la reunión de CEPI-COVID-19, Médicos sin Fronteras publicó una carta abierta dirigida a la junta directiva de CEPI, solicitándole reafirmar sus compromi-

24 Coy, P., "Pay Attention to Nobel Laureate Michael Kremer on the Pandemic", Bloomberg Businessweek, 6 de mayo de 2020, disponible en: https:/wwrw.bloomberg.com/news/ articles/2020-05-06/pay-attention-to-nobel-laureate-michael-kremer-on-the-pandemic.

25 Newey, S., "China participates in global coronavirus summit while US maintains silences", The Telegraph, 4 de mayo de 2020, disponible en: https://wreretelegraph.co.uk/globalhealth/science-and-disease/china-participates-global-coronavirus-summit-us-maintains-silence/; Booth, W., Johnson, C., Morello, C. "The world came together for a virtual vaccine summit. The U. S. was conspicuously absent", The Washington Post, 4 de mayo de 2020, disponible en: https:// wwwewashingtonpost.com/world/europe/the-world-comes-together-for-a-virtual-vaccine-summit-the-us-isconspicuously-absent/2020/05/04/ac5b6754-8a5c-11ea-80df-d24b35a568ae_story.html. 
Este libro forma parte del acervo de la Biblioteca Jurídica Virtual del Instituto de Investigaciones Jurídicas de la UNAM

sos de garantizar un acceso efectivo y equitativo a las vacunas producidas con sus fondos. Médicos sin Fronteras decidió publicar esa nota luego de que CEPI realizara cambios en su política de acceso equitativo a vacunas. En su versión original el documento explicaba los términos definidos por CEPI que debían acatar las empresas e instituciones que recibieran los fondos para investigación y desarrollo de vacunas. Estos términos abarcaban desde la fijación de precios hasta las políticas de propiedad intelectual. En la versión resumida que circuló, todos esos detalles se omitieron. Para Médicos sin Fronteras ese cambio no es menor y generó alertas. Sin embargo, la reacción de la CEPI frente a la nota de Médicos sin Fronteras ha sido mantener esa última versión de su política de acceso. Aquí hay un extracto de la carta:

La Política de Acceso Equitativo revisada de CEPI constituye un alarmante paso atrás para la organización porque ya no garantiza que las vacunas producidas a través de los fondos del CEPI estarán disponibles a un precio asequible. Tampoco proporciona ninguna base para que CEPI rinda cuentas a sus inversores públicos y filantrópicos en sus acuerdos con socios de desarrollo sobre quién tendrá la titularidad y cuál será el tratamiento de la propiedad intelectual o cómo se determinará el precio de las vacunas financiadas por CEPI. Al reemplazar la política de acceso equitativo previamente detallada con solo una declaración de principios amplia, la política revisada marca un giro preocupante que se aleja de los compromisos iniciales de CEPI con el acceso, la transparencia y la apertura, y abre nuevos caminos en términos de responsabilidad pública. ${ }^{26}$

Para quienes han investigado desde la academia y desde organizaciones de derechos humanos y acceso a medicamentos, acerca del impacto que el régimen global de producción de medicamentos ha tenido en la salud pública, como Ellen t' Hoen, de Medicines, Law and Policy y asesora del fondo de UNITAID para la producción de medicamentos para la infección por HIV, y Merith Basey, directora norteamericana de Universities Allied for Essential Medicines, el rechazo de las grandes transnacionales farmacéuticas a los términos de garantía de precios de CEPI era algo esperable. Enfatizan que toda empresa farmacéutica que esté recibiendo fondos de agencias internacionales en esta, o en cualquier otra crisis, debe rendir cuentas públicamente y están en la obligación de divulgar los términos y

26 Mèdecin sans Frontières, Open letter to CEPI Board Members: Revise CEPI's access policy, 5 de marzo de 2020, disponible en: https://wrewe.msfaccess.org/open-letter-cepi-boardmembers-revise-cepis-access-policy. Traducción propia. 
Este libro forma parte del acervo de la Biblioteca Jurídica Virtual del Instituto de Investigaciones Jurídicas de la UNAM

condiciones de los acuerdos de colaboración entre los Estados, las agencias y las empresas. ${ }^{27}$

Desde una perspectiva geopolítica, ${ }^{28}$ los medicamentos y las vacunas para COVID-19 tienen un enorme valor estratégico, más allá de los intereses nacionalistas, que puede cambiar el lugar que ocupan los diversos actores globales en la disputa por la hegemonía económica, política y cultural. Ya es obvio que la competencia es feroz, sobre todo entre EEUU, Rusia y China. La competencia no se limita al financiamiento de investigaciones, sino que además involucra otras acciones comerciales y políticas, algunas de las cuales han sido vistas como gestos de hostilidad. A manera de ilustración, podemos ver el intento del gobierno de los EEUU, hace unos meses, por comprar una empresa desarrolladora de vacunas alemana. Este caso provocó gran indignación en Europa porque, de lograrse ese acuerdo comercial, la empresa habría privilegiado el mercado estadounidense. Otro gesto político y comercial que permite darse una idea de la agresividad del juego geopolítico en el contexto de la actual crisis global, es la compra que hizo el gobierno de los EEUU a la compañía farmacéutica Gilead por el total de su producción de remdesivir. ${ }^{29}$

\section{JUSTICIA Y ACGESO A TEGNOLOGÍAS EN TIEMPOS DE PANDEMIA: ENTRE CIENCIA ABIERTA Y CIENCIA GERRADA}

Diversos autores y centros de investigación en bioética han realizado numerosas publicaciones advirtiendo a los Estados, instituciones sanitarias y a la amplia gama de sectores sociales, acerca de la relevancia fundamental de po-

27 Zaitchik, Alexander, "No Vaccine in Sight. The U. S. was once at the cutting edge of pandemic prevention. Then Big Pharma took over", The New Republic, 11 de mayo de 2020, disponible en: https://newrepublic.com/article/157594/no-coronavirus-vaccine-big-pharma-drug-patent-system.

28 Moon, S. "The Vaccine Race: Will Public Health Prevail over Geopolitics?", The Graduate Institute of Geneva. Global Challenges. Special Issue núm. 1, junio de 2020, disponible en: https://globalchallenges.ch/issue/special_1/the-vaccine-race-will-public-health-prevail-overgeopolitics/; Bremmer, I. "What Vaccine Nationalism Means for the Coronavirus Pandemic", Time, 27 de julio del 2020, disponible en: https://time.com/5871532/vaccine-nationalism-coronavirus-pandemic/.

29 Boseley, S., "US secures world stock of key Covid-19 drug remdesivir", The Guardian, 30 de junio de 2020, disponible en: https://wrwetheguardian.com/us-news/2020/jun/30/ us-buys-up-world-stock-of-key-covid-19-drug; Martin, Ian. "U. S. Buys The World's Supply of Breakthrough Coronavirus Drug Remdesivir", Forbes, 1o. de julio del 2020, disponible en: https://wrere:forbes.com/sites/iainmartin/2020/07/01/us-buys-the-world-supply-of-breakthrough-coro navirus-drug-remdesivir/\#4eb17f545472. 
Este libro forma parte del acervo de la Biblioteca Jurídica Virtual del Instituto de Investigaciones Jurídicas de la UNAM

líticas de justicia distributiva para asignar de manera justa los escasos recursos (sean cuales fuere) durante esta emergencia global. Las preguntas sobre cómo asignar de la manera más justa posible los diversos bienes y servicios indispensables para enfrentar la pandemia van desde cómo priorizar el uso de los medicamentos, las camas de cuidados intensivos, los equipos de ventilación mecánica y hasta cómo determinar el uso más efectivo de las pruebas diagnósticas, la distribución de los equipos de protección personal y, por supuesto, quiénes deberían ser los primeros en recibir una eventual vacuna contra el SARS-GoV-2.30

Los gobiernos deben ser transparentes frente a la población con respecto a las decisiones y estrategias de priorización para la asignación de tratamientos, vacunas y demás recursos. Deben comunicarse los valores y principios en los cuales se enmarca la política pública y deben explicar cómo se derivan las rutas de toma de decisión de dichos principios. El mismo principio aplica para los organismos internacionales, consorcios y alianzas público-privadas en sus funciones atinentes a financiar, promocionar y facilitar la investigación, desarrollo y manufactura de bienes críticos para la respuesta a la pandemia por COVID-19. Deben divulgar los razonamientos éticos y políticos en los cuales se fundamentan las decisiones relacionadas con cualquier aspecto de atención de la pandemia.

Estas son aspiraciones éticas que, en realidad, no son novedosas, sino que responden a un largo recorrido de análisis, activismo y diplomacia en favor del derecho humano a la protección de la salud y del derecho humano al goce de los avances científicos y tecnológicos. No obstante, la realidad se impone y una vez más, estamos presenciando esa tensión que parece nunca acabar, entre los derechos de propiedad intelectual y la protección de la salud pública. ${ }^{31}$

Es precisamente por ese constante conflicto entre propiedad intelectual y salud pública que cobra especial relevancia la argumentación de Henry

30 Kohler, J. y Mackey, T., "Why the COVID-19 pandemic should be a call for action to advance equitable access to medicines. Fair and equitable access to COVID-19 treatments and vaccines", Nuffield Council of Bioethics, 29 de mayo de 2020; Berlinger, N. et al., "Ethical framework for health care institutions responding to novel coronavirus SARS-CoV-2 (COVID-19). Guidelines for institutional ethics services responding to COVID-19", The Hastings Center, 16 de marzo de 2020, disponible en: https://wrwe.thehastingscenter.org/wp-content/ uploads/HastingsCenterCovidFramework2020.pdf.

31 Bonadio, E. y Baldini, A., "COVID-19, Patents and the never-ending tension between proprietary rights and the protection of public health", European Fournal of Risk Regulation, 2020, pp. 1-6; Ravelo, J., "Battle over CEPI's access to vaccines policy deepens", Devex, 11 de marzo de 2020, disponible en: https://wrew.devex.com/news/battle-over-cepi-s-access-to-vaccinespolicy-deepens-94438. 
Este libro forma parte del acervo de la Biblioteca Jurídica Virtual del Instituto de Investigaciones Jurídicas de la UNAM

Chesbrough, ${ }^{32}$ director del Garwood Center, en la Universidad de Berkeley, en su artículo académico "To recover faster from COVID-19, open up", publicado en la revista Industrial Marketing Management.

Como he explicado a lo largo de este capítulo, la investigación científica y la innovación tecnológica son la mayor fuente de esperanza para el mundo, en estos momentos de grave crisis sistémica. Después de meses en los que miles de personas, en el mundo, han muerto por las complicaciones del COVID-19, millones han tenido que soportar la tragedia de perder a un ser querido, de verse forzadas a vivir en algún grado de confinamiento muchas veces sin tener las más mínimas condiciones materiales para ello-, han perdido sus trabajos o sus pequeños negocios o han sobrevivido la enfermedad, pero están experimentando serias y complejas secuelas en su salud, el mundo entero espera ansioso la noticia de que ya existe una vacuna o un medicamento que puede controlar la infección o, mejor aún, prevenirla.

Si bien la solución a los problemas de injusticia estructural que han potenciado los diversos y graves efectos de esta pandemia no se solucionarán mágicamente con intervenciones tecnológicas, es irrefutable que sin una gama de tecnologías no será posible superar, en el mediano plazo, esta crisis generalizada. El argumento del profesor Chesbrough no es novedoso, pero es certero y pertinente: para producir todas las tecnologías (incluyendo medicamentos y vacunas) que el mundo necesita para superar la pandemia, la ruta es abrir la investigación científica y la innovación.

Esto implica levantar, suspender o eliminar toda forma de barrera de acceso a la información, datos, diseños, en fin, a todo conocimiento vital para los procesos de desarrollo científico y tecnológico, que son críticos para responder a las diversas necesidades sanitarias en esta pandemia. En otras palabras, si como sociedad global queremos superar la pandemia lo antes posible, la apuesta de los Estados y de las organizaciones intergubernamentales debe ser por la ciencia y la innovación abiertas.

Para concluir, quiero traer a colación uno de los documentos más importantes en la defensa de la ciencia abierta: el pronunciamiento "Who owns science? The Manchester Manifesto", ${ }^{33}$ publicado en el 2010, bajo el auspicio y liderazgo de John Sulston y Joseph Stiglitz. Este documento revisa los conflictos relativos al uso de la propiedad intelectual en el mun-

32 Chesbrough, H., "To recover faster from Covid-19, open up: Managerial implications from an open innovation perspective", Industrial Marketing Management, núm. 88, 2020, pp 410-413.

33 Sulston, John et al., Who owns science? The Manchester Manifesto, University of Manchester, 2012. 
Este libro forma parte del acervo de la Biblioteca Jurídica Virtual del Instituto de Investigaciones Jurídicas de la UNAM

do tecnocientífico y problematiza el concepto de apropiación de la ciencia. A su vez, cuestiona el modo en que el sistema de propiedad intelectual ha permeado en la cultura científica, favoreciendo más la competencia (con propósitos de maximización de ganancias comerciales) que la colaboración y divulgación del conocimiento. En este sentido la propuesta de Costa Rica es coherente con un llamado a la reflexión dentro del campo científico-tecnológico, acerca de las implicaciones que la cultura de la competencia y el secretismo en la ciencia tiene sobre el desarrollo de la ciencia misma y sobre los derechos humanos de las personas en todo el mundo.

La actual crisis global puede ser el momento crítico que nos lleve de vuelta a modelos de ciencia más abiertos y colaborativos, orientados por principios éticos de justicia, equidad, transparencia e inclusión.

\section{CONCLUSIONES}

A diferencia de otros bienes que se transan en el mercado, el conocimiento es un bien inagotable. Por lo tanto, no es necesario restringir el acceso al conocimiento. Para proteger o gestionar otros bienes que sí son finitos, es posible encontrar justificaciones razonables para controlar el acceso y uso a esos bienes, porque un uso irracional o inequitativo podría agotar rápidamente ese bien, para perjuicio de toda la población. Sin embargo, en el caso del conocimiento científico, ocurre lo contrario. El acervo mundial de conocimiento aumenta conforme más personas tienen acceso a él y colaboran en la divulgación, aplicación y creación de más conocimiento.

En una situación límite y extremadamente compleja, como la actual pandemia, la colaboración es más efectiva que la competencia para proveer soluciones efectivas y seguras en el menor tiempo posible. Sin embargo, los intereses comerciales y geopolíticos han prevalecido una vez más. Pero la realidad se impondrá. Sin justicia distributiva la pandemia no estará superada en ninguna parte del mundo, porque una pandemia no está controlada hasta que el más vulnerable de los países haya logrado contenerla. 